\title{
Archives
}

\section{Diffuser, partager et s'approprier le patrimoine documentaire québécois. Le projet collaboratif de BAnQ sur Wikimédia : une première au Canada}

\author{
Denis Boudreau, Florian Daveau et Frédéric Giuliano
}

Volume 46, numéro 1, 2016

URI : https://id.erudit.org/iderudit/1035723ar

DOI : https://doi.org/10.7202/1035723ar

Aller au sommaire du numéro

Éditeur(s)

Association des archivistes du Québec (AAQ)

ISSN

0044-9423 (imprimé)

2369-9256 (numérique)

Découvrir la revue

Citer cet article

Boudreau, D., Daveau, F. \& Giuliano, F. (2016). Diffuser, partager et s'approprier le patrimoine documentaire québécois. Le projet collaboratif de BAnQ sur Wikimédia : une première au Canada. Archives, 46(1), 61-81. https://doi.org/10.7202/1035723ar
Résumé de l'article

Les trois auteurs font état de leur expérience et des stratégies mises en oeuvre dans la réalisation du projet collaboratif de Bibliothèque et Archives nationales du Québec (BAnQ) sur Wikimédia Commons. Ce projet vise à assurer la réalisation d'une des plus importantes missions que s'est donnée BAnQ : offrir un accès démocratique à la culture et à la connaissance.

Le projet s'articule autour de quatre volets. Le premier, lancé en 2013, porte sur l'ajout de liens vers des fonds d'archives à plus de 800 articles sur l'encyclopédie collaborative Wikipédia. À l'hiver 2014, un second volet, portant cette fois sur la formation des usagers de Wikipédia, est mis en branle. Le projet Mardi c'est Wiki !, gratuit et ouvert à tous, vise à améliorer le contenu francophone de l'encyclopédie en formant les contributeurs. Le troisième volet, le projet Poirier, débute pour sa part à l'été 2014 et se veut un outil pour la sélection et la mise en ligne de centaines de photographies libres de droit sur Wikimédia. Enfin, le quatrième volet, lancé à l'automne 2014, porte sur la transcription collaborative d'ouvrages conservés par BAnQ sur Wikisource, un projet de bibliothèque numérique collaborative.

Du point de vue des auteurs, ce projet et les volets qui en découlent sont des outils qui permettent le partage du patrimoine et une collaboration élargie du public. Cette participation active de BAnQ à Wikimédia Commons favorise grandement la visibilité de ses collections et, par le fait même, leur diffusion.
Ce document est protégé par la loi sur le droit d'auteur. L’utilisation des services d'Érudit (y compris la reproduction) est assujettie à sa politique d'utilisation que vous pouvez consulter en ligne.

https://apropos.erudit.org/fr/usagers/politique-dutilisation/ 


\section{NOTE ET BILAN D'EXPÉRIENCE}

\section{Diffuser, partager et s'approprier le patrimoine documentaire québécois.}

Le projet collaboratif de BAnQ sur Wikimédia: une première au Canada

\section{Denis Boudreau \\ Florian Daveau Frédéric Giuliano}

L'une des missions fondamentales de Bibliothèque et Archives nationales du Québec (BAnQ) est d'offrir un accès démocratique à la culture et à la connaissance. Tous ou presque connaissent la Grande Bibliothèque, élément phare de l'institution ayant su s'imposer dans le paysage culturel québécois. Mais BAnQ, c'est aussi 11 autres établissements répartis à la grandeur de la province grâce auxquels elle peut déployer ses activités. Les fonds et les collections dont BAnQ est responsable sont vastes et diversifiés. On y trouve tout autant les archives de l'État que des fonds d'archives photographiques, des manuscrits d'écrivains, des films, des disques, des partitions musicales, des affiches, des programmes de spectacles, des estampes, des livres d'artistes, des cartes postales et des cartes géographiques, sans oublier pratiquement tous les livres, journaux et revues publiés au Québec depuis 1764, des millions de documents qui constituent en quelque sorte la mémoire du Québec.

Archivistes et bibliothécaires ne font pas que rassembler passivement ce vaste ensemble patrimonial. Ils travaillent d'arrache-pied pour qu'il soit mieux connu et plus facilement accessible aux Québécois. Ainsi, BAnQ accueille donc dans ses murs toute personne sans distinction aucune. Elle offre plus particulièrement ses services aux citoyens du Québec, aux chercheurs, aux utilisateurs de services bibliographiques, 
aux gens du milieu de l'édition, aux représentants des institutions documentaires et culturelles locales, régionales, nationales ou étrangères et aux autres professionnels des milieux documentaires, ainsi qu'aux représentants des ministères et des organismes gouvernementaux. En priorité, les efforts de BAnQ sont consacrés à augmenter le contenu des collections numériques, meilleur moyen d'atteindre une population répartie sur un immense territoire. Mais d'autres initiatives ont aussi été menées au cours des dernières années pour établir une médiation active et fluide avec le public: création de blogues et d'une foire aux questions, géolocalisation progressive des collections, déploiement d'un programme d'ateliers-conférences, création d'expositions et de circuits thématiques virtuels, etc. Et depuis juin 2013, une nouvelle étape importante a été franchie dans le décloisonnement des collections par la mise sur pied d'une fructueuse collaboration avec Wikimédia Canada, association grâce à laquelle BAnQ dispose désormais d'outils éprouvés pour solliciter la participation citoyenne à sa mission de diffusion du patrimoine québécois.

\section{PRÉSENTATION DU PROJET}

Lancé au cours de l'été 2013, le projet de BAnQ dans l'univers Wikimédia est une première au Canada ${ }^{1}$. Wikimédia est un mouvement mondial qui s'est donné pour mission de rendre disponible du contenu éducatif gratuitement à l'ensemble de la population. Il se décline en de nombreux projets (notamment Wikipédia) où chaque individu peut participer librement à la somme des connaissances. Le projet de BAnQ s'articule en plusieurs volets annoncés sur sa nouvelle page GLAM (acronyme pour Galleries, Librairies, Archives and Museums). Cet acronyme désigne plus généralement des institutions publiques chargées de la préservation et de la diffusion du patrimoine culturel et historique, actives sur les plateformes de Wikimédia. Hébergée directement sur le site de Wikipédia, BAnQ a développé cette page qui sert à la fois de vitrine présentant les projets de l'institution dans l'univers Wikimédia et de lieu d'échange entre les contributeurs et l'institution.

Fondé en 2001, Wikipédia est le site de la famille Wikimédia le plus populaire. Encyclopédie collaborative multilingue, ce site contient 1695000 articles en français et 29 millions d'articles toutes langues confondues (novembre 2015). Depuis sa création, Wikipédia a reçu plus de 100 milliards de visites; en 2015, il figurait au septième rang des sites les plus consultés au monde ${ }^{2}$.

Alors que Wikipédia était perçu avec méfiance à ses débuts par les professionnels des sciences de l'information, bon nombre d'entre eux en sont venus à l'intégrer au sein de leurs processus de référence. Étant donné la popularité croissante de l'encyclopédie, un consensus semble se former sur son utilité, les professionnels soulignant tout de même la nécessité d'une approche critique et d'une bonne compréhension de sa nature. Dans ce contexte, Wikipédia devient non seulement un outil de référence et de formation à la littératie numérique (Calhoun 2014, 32), mais également un formidable moyen pour mettre en valeur des ressources auprès du grand public. Le fort potentiel de diffusion de Wikipédia et le fait que sa mission de démocratisation du savoir soit en accord avec celle de BAnQ nous ont confortés dans notre décision de collaborer avec Wikimédia. 


\section{|BAIII}

GLAM (i) WIKI

\begin{tabular}{l|l|l|l|l|l|l} 
A & $\begin{array}{l}\text { COMMONS: } \\
\text { PROJET POIRIER }\end{array}$ & $\begin{array}{l}\text { WIKISOURCE:PROJET } \\
\text { DE TRANSCRIPTION }\end{array}$ & $\begin{array}{l}\text { WIKISOURCE:LIVRES } \\
\text { DE RECETTES }\end{array}$ & $\begin{array}{l}\text { WIKIPÉDIA: } \\
\text { MARDI C'EST WIKI }\end{array}$ & $\begin{array}{l}\text { WIKIPÉDIA: } \\
\text { PROJET:QUÉBEC }\end{array}$ & $\begin{array}{l}\text { POSER UNE QUESTION } \\
\text { AUX BIBLIOTHÉCAIRES }\end{array}$
\end{tabular}
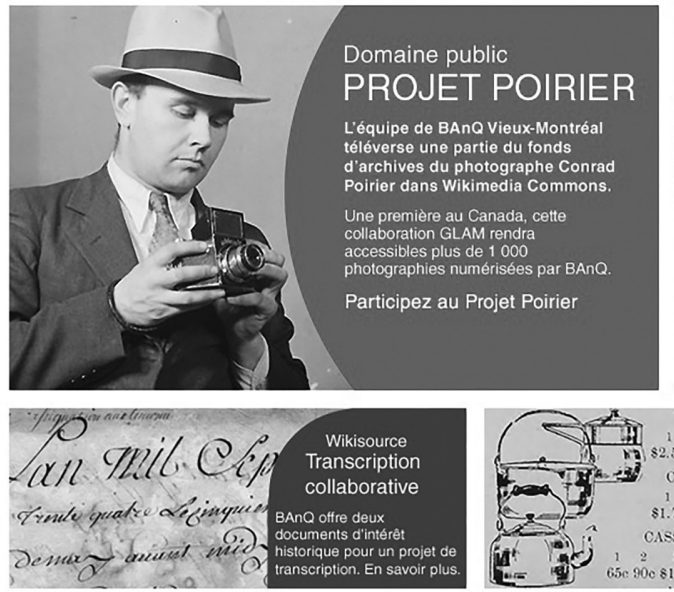

Bienvenue sur la page GLAM de Bibliothèque et Archives nationales du Québec (BAnQ). Cette page rassemble les différents projets Wiki auxquels prend part l'institution.

Depuis janvier 2014, BAnQ, Wikimédia Canada et divers partenaires collaborent afin d'offrir aux usagers de BAnQ plusieurs activités en lien avec les projets Wikimédia.

Ces projets visent à mettre en valeur les fonds et les collections de $B A n Q$, à rendre disponibles aux wikimédiens les ressources numérisées de l'institution et à contribuer à l'enrichissement des articles de Wikipédia (et ses projets frères) concernant le Québec, le Canada français et, plus largement, l'Amérique française.
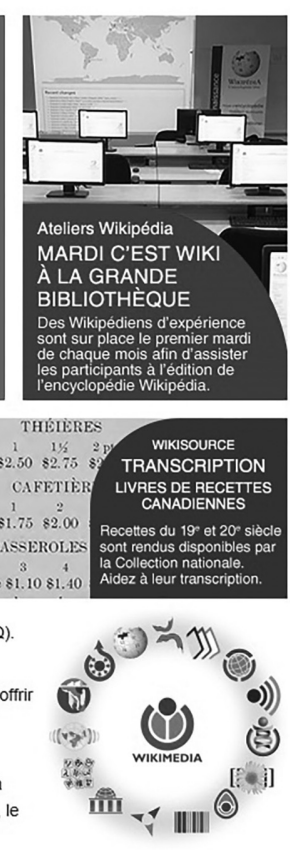

Actualités

Samedi c'est Wiki à BAnQ ! Atelier de formation à Wikipédia le samedi 26 septembre de 13 à $16 \mathrm{~h}$ dans le cadre de la linformatique libre of (SQIL)

Livres de recettes La Collection nationale de recettes du 19 e trois livres de en vue de leur transcription. Projet Wikisource BAnQ a téléversé deux nouveaux documents en vue de leur transcription. Projet Poirier
Á rété 2014, réquipe de BAnQ Vieux-Montréal a entrepris de téeverser dans le repertoire de medias libres Wikimedia
Commons, plusieurs centaines d'images de Montréal et de ses environs.

Mardi, c'est Wiki! à BAnQ Depuis février 2014, BAnQ et Wikimédia organisent des ateliers gratuits afin de former les gens sintéressant au processus d'édition de rencyclopédie en ligne Wikipédia. Le premier mardi de chaque mois, des Wikipédien pour guider les participants.

)) Liens rapides

- Mardi, c'est Wiki! à la

- Projet Poirier

- Portail Nouvelle-France de Wikisource

- Les archives à BAnQ ש

Figure 1 - Aperçu de la page GLAM de BAnQ. (https://fr.wikipedia.org/wiki/Wikipédia :BAnQ)

Un premier volet, lancé à l'été 2013, consiste à collaborer à l'amélioration du contenu québécois de l'encyclopédie Wikipédia. Notre intervention a porté sur l'ajout d'une mention indiquant l'existence d'un fonds d'archives et son lieu de conservation dans plus de 800 articles de l'encyclopédie. Un lien menant vers la notice descriptive du fonds d'archives dans Pistard (le moteur de recherche des documents d'archives de BAnQ) a aussi été ajouté.

Un second volet lancé en février 2014, Mardi, c'est Wiki!, se concentre sur l'aspect formation des utilisateurs de Wikimédia. Le premier mardi de chaque mois, des "wikipédiens" d'expérience et des employés de BAnQ partagent lors de ces ateliers leurs connaissances avec tous ceux qui désirent en savoir plus sur l'encyclopédie et ses projets frères. Il s'agit de la première activité de formation de ce genre proposée au sein d'une institution patrimoniale au Canada. Présentée en collaboration avec Wikimédia Canada à la Grande Bibliothèque, cette activité, gratuite et ouverte à tous, vise à améliorer le contenu francophone de Wikipédia, à augmenter le nombre de contributeurs québécois ainsi qu'à tirer profit des ressources documentaires et professionnelles de BAnQ. Elle 
a également pour but de mieux faire connaître le Québec, la Nouvelle-France, le Canada français et, plus largement, l'Amérique française. Lors de cette activité à laquelle participent la Fondation Lionel-Groulx et l'Association francophone pour le savoir (Acfas), l'animateur rappelle l'importance de citer des sources fiables, de multiplier les points de vue et de croiser les informations.

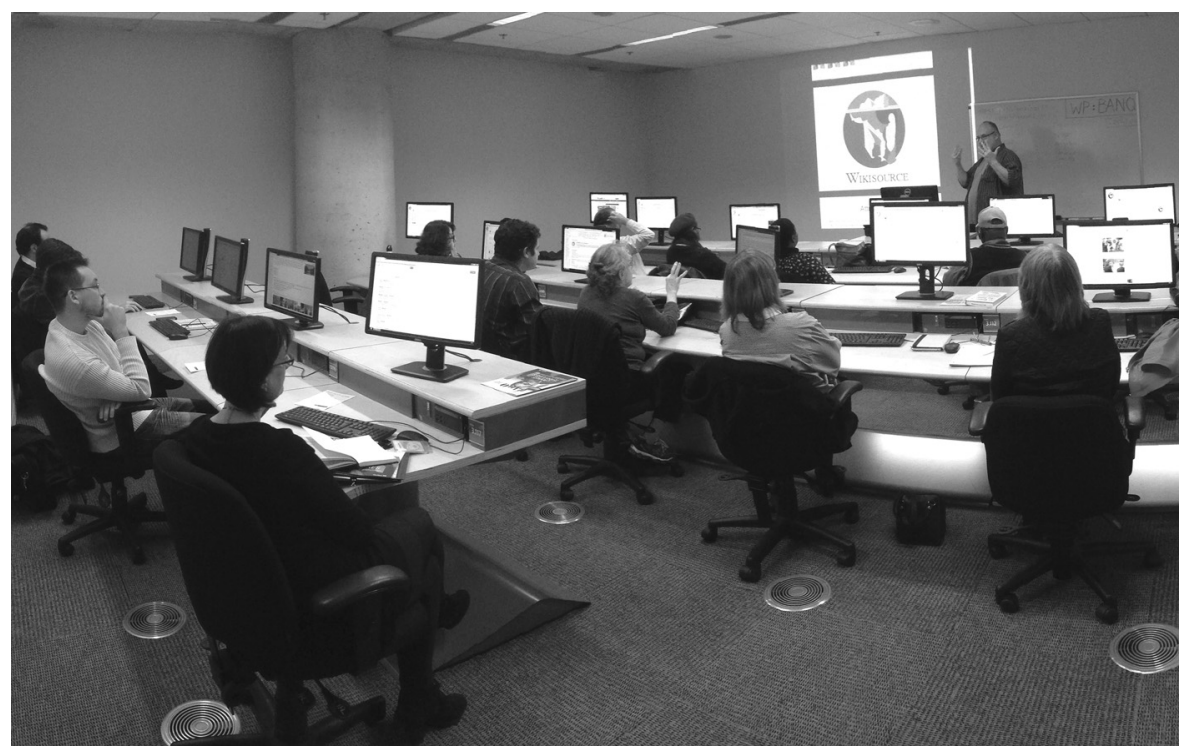

Figure 2 - Mardi, c'est wiki! à la Grande Bibliothèque, le 19 octobre 2014. Photo: Benoit Rochon.

Des bibliothécaires et des archivistes guident les participants dans les ressources numériques, modernes et anciennes, qui s'offrent à eux. Et, pour poursuivre leurs travaux entre deux ateliers, les abonnés de BAnQ bénéficient d'un prêt prolongé de documents. De plus, il est possible pour certains citoyens de suivre les séances en direct et de collaborer à distance ${ }^{3}$.

Le troisième volet, appelé projet Poirier, amorcé à l'été 2014, est davantage centré sur la participation active des contributeurs pour alimenter le contenu des articles de l'encyclopédie Wikipédia. Pionnière en la matière au Canada, BAnQ a sélectionné et téléversé des centaines de photographies dans Wikimedia Commons, projet frère de Wikipédia, qui se définit comme "une médiathèque de [...] fichiers média librement réutilisables et que chacun peut enrichir " ${ }^{4}$. Véritable bibliothèque d'images regroupant des cartes, des photographies et des dessins, Commons héberge également des films et des enregistrements sonores qui peuvent par la suite être ajoutés dans plusieurs articles de Wikipédia, dans toutes les langues, multipliant ainsi le potentiel de diffusion d'un seul document. L'objectif de ce volet est de mettre à la disposition des contributeurs de Wikipédia des centaines d'images du patrimoine qu'ils peuvent librement utiliser pour illustrer des articles des différentes versions linguistiques de l'encyclopédie. Les contributeurs sont invités à insérer les images dans des articles et à les indexer dans la banque d'images pour faciliter leur repérage. Cette démarche offre également un 
nouvel accès au patrimoine documentaire québécois et, plus largement, à la culture québécoise en proposant du contenu patrimonial sur une plateforme consultée par le grand public.

Enfin, le quatrième volet, lancé à l'automne 2014, implique la transcription collaborative d'ouvrages des collections de BAnQ sur Wikisource. Cet outil en ligne est en fait un projet de bibliothèque numérique multilingue élaborée par des contributeurs bénévoles dont l'objectif est d'inviter la communauté "wikimédienne" à participer à la création, à la transcription ou à la relecture d'un ouvrage ou de documents d'archives, tout en respectant les règles du droit d'auteur et celles établies par la communauté. Ces deux derniers volets représentent des pas significatifs en matière de développement d'un esprit collaboratif entre BAnQ et ses usagers puisque le contenu proposé peut être bonifié, commenté et corrigé librement. Cet article présentera plus en détail la méthodologie et les résultats de deux volets qui ont mis en lumière l'importance pour BAnQ de s'inscrire comme acteur de l'univers Wikimédia : la contribution directe aux articles par l'ajout de références et le projet Poirier.

\section{PERTINENCE DES NOTICES ARCHIVISTIQUES DANS LES SOURCES DE WIKIPÉDIA}

Wikipédia est un outil d'autant plus utile en raison de son libre accès, de ses citations fréquentes de contenu, de ses ressources pertinentes ainsi que de son ouverture à la participation de tout un chacun (Murley 2008, 593-607). Comme le souligne West et Williamson :

Il semblerait que le perpétuel débat entre professionnels [des sciences de l'information] au sujet des avantages et inconvénients de Wikipédia n'est pas pertinent. L'encyclopédie existe, elle est largement utilisée. Elle est un point de départ logique pour la recherche pour plusieurs individus; elle est particulièrement utile en tant qu'outil de mise en contexte pour établir les fondements d'une recherche plus approfondie [...] Wikipédia a sa place au sein du processus de recueillement d'information. (West et Williamson, notre traduction)

En considérant la nature et l'étendue de ses contenus, Wikipédia est non seulement l'un des plus grands portails Internet consacré à l'histoire et à la culture, c'est aussi un important dépôt de connaissances à la disposition des acteurs du monde des sciences de l'information, de la formation et de l'enseignement (Snyder 2013, 155). On y trouve une plateforme idéale pour la mise en valeur de fonds d'archives par l'entremise de ses articles de nature historique, consacrés à des personnages ou à des institutions de toute nature (Rosenweig 2006, 119). Ce potentiel est d'autant plus pertinent que le repérage des contenus "wikipédiens" est grandement facilité par des moteurs de recherche tels que Google ou Bing qui leur accordent une place importante dans leurs algorithmes de recherche (Doucet Rand 2010, 924; Barron 2011, 12-16).

Constamment positionné depuis plusieurs années au sein des dix sites Web les plus consultés dans le monde et très utilisé par les étudiants et les chercheurs, Wikipédia peut dans les faits servir de passerelle vers nos ressources archivistiques au début de leur recherche (Dumouchel et Karsenti 2012). Ainsi, nous nous insérons dans les habitudes de recherche des internautes qui sont tout autant d'usagers en devenir de 
nos ressources et par conséquent, tout autant d'agents multiplicateurs de la notoriété du patrimoine culturel québécois.

\section{Démarche et résultats}

$\mathrm{Au}$ moment du lancement de ce premier volet, c'est 3030 fonds d'archives privées qui doivent faire l'objet d'une vérification dans l'encyclopédie. Nous avons limité notre démarche à la version francophone de Wikipédia. Lorsqu'un article est répertorié, une courte phrase et un hyperlien vers la notice du fonds d'archives sont insérés dans l'article. Ainsi peut-on lire dans l'article consacré à Lionel Groulx : "Le fonds d'archives Lionel Groulx (CLG1) est conservé au centre BAnQ Vieux-Montréal de Bibliothèque et Archives nationales du Québec". Ensuite, dans la section des références, il a été ajouté : Fonds Lionel Groulx (CLG1) - Bibliothèque et Archives nationales du Québec (BAnQ).", sur Pistard - Bibliothèque et Archives nationales du Québec (consulté le 25/09/2014); et l'hyperlien vers la notice descriptive dans Pistard. Les problématiques et particularités rencontrées sont consignées dans un tableau de suivi. Un guide est aussi préparé pour assister le personnel affecté à la tâche. Ce document présente les consignes et la marche à suivre pour l'insertion de texte dans Wikipédia.

Au final, c'est plus du quart des fonds répertoriés qui donnent lieu à une intervention dans Wikipédia.

Tableau 1 - Bilan statistique par centre d'archives (31 janvier 2014)

\begin{tabular}{|l|c|c|}
\hline Centres & $\begin{array}{c}\text { Nombre de fonds privés } \\
\text { recensés }\end{array}$ & $\begin{array}{c}\text { Nombre d'articles Wikipédia } \\
\text { pertinents }\end{array}$ \\
\hline BAnQ Vieux-Montréal & 1377 & $522(38 \%)$ \\
\hline BAnQ Québec & 707 & $217(31 \%)$ \\
\hline BAnQ Gatineau & 216 & $12(3 \%)$ \\
\hline BAnQ Sherbrooke & 61 & $20(13 \%)$ \\
\hline BAnQ Saguenay & 160 & $18(13 \%)$ \\
\hline BAnQ Trois-Rivières & 142 & $8(15 \%)$ \\
\hline $\begin{array}{l}\text { BAnQ Rimouski } \\
\text { (incluant BAnQ Gaspé) }\end{array}$ & 54 & $17(6 \%)$ \\
\hline BAnQ Rouyn-Noranda & 254 & $7(12 \%)$ \\
\hline BAnQ Sept-Îles & 59 & $826(27 \%)$ \\
\hline \multicolumn{1}{c|}{ Totaux } & 3030 & \\
\hline
\end{tabular}

Tableau 2 - Bilan statistique par classe de fonds d'archives (31 janvier 2014)

\begin{tabular}{|l|c|c|}
\hline Classes $^{5}$ & Nombre de fonds recensés & $\begin{array}{c}\text { Nombre d'articles Wikipédia } \\
\text { pertinents }\end{array}$ \\
\hline CLG & 67 & $32(48 \%)$ \\
\hline MSS & 463 & $248(54 \%)$ \\
\hline P & 2500 & $546(22 \%)$ \\
\hline & 3030 & $826(27 \%)$ \\
\hline
\end{tabular}


Nous constatons qu'il existe une grande disparité entre les fonds d'archives selon la région de leur provenance. Si les fonds localisés à Montréal peuvent être jumelés à un contenu "wikipédien" dans près de $40 \%$ des cas et $30 \%$ des cas pour les fonds de Québec, ce pourcentage chute pour les fonds d'archives conservés dans les autres centres de BAnQ (entre 3\% et 18\%, selon le cas). Ces disparités s'expliquent en partie par la nature communautaire de cette encyclopédie, la création d'articles étant surtout motivée par des considérations géographiques, par les intérêts des participants et par les principaux acteurs de l'actualité. Il demeure cependant difficile de quantifier l'impact de ces interventions quant à la consultation et l'utilisation de nos ressources, si ce n'est que plusieurs usagers nous ont mentionné avoir pris connaissance de l'existence d'un fonds d'archives suite à la consultation de Wikipédia. Néanmoins, l'acquisition de nouveaux fonds d'archives fait désormais l'objet d'une vérification et le cas échéant d'un ajout dans Wikipédia.

\section{La communauté "wikipédienne"}

Au sein de cette communauté, tout un chacun peut rédiger et éditer un article. On y trouve par contre toute une série de mesures qui favorisent l'encadrement des nouveaux contributeurs et qui permettent le signalement d'abus, d'erreurs et de mauvaises pratiques. Aussi, bien que la majorité de nos interventions dans les articles aient été réalisées sans problème et n'aient pas fait l'objet de commentaires particuliers, le bien-fondé de notre démarche a été rapidement questionné et accueilli avec scepticisme. La réaction initiale de la communauté, dans les premiers moments de notre travail, nous a surpris ${ }^{6}$. Un grand nombre de commentaires démontrent une réelle méconnaissance de la nature et de l'utilité des documents d'archives dans la formation d'un savoir collectif. Aussi, la publication d'une réponse présentant l'objectif de notre démarche a été très bien accueillie.

En parcourant les forums ${ }^{7}$, on constate que ces intervenants utilisent un grand nombre de ressources provenant de BAnQ dans leurs travaux d'édition et de rédaction? De plus, des commentaires nous permettent de constater que la mise en place par BAnQ de moyens pour faciliter l'utilisation de ces ressources dans les textes de Wikipédia est fortement souhaitée. Avec le recul, nous estimons que davantage d'attention aurait été nécessaire pour se familiariser avec la culture et la communauté "wikipédienne" afin d'en saisir les codes et les usages. Une présentation de notre projet dans les forums de discussions de l'encyclopédie aurait sans doute été bénéfique, solution transparente qui a été privilégiée lors des autres projets.

Ainsi, l'importance et la nature de la communauté "wikipédienne" ne sont pas non plus à négliger. En effet, comment les bibliothèques et les dépôts d'archives pourraient-ils tourner le dos à cette masse d'individus passionnés par l'acquisition et le partage d'information? Comme le souligne Peter Binkley: "Bien que n'ayant pas officiellement d'éditeurs, la communauté "wikipédienne" nous offre l'avantage d'être composée d'usagers idéals. C'est-à-dire des personnes passionnées par l'acquisition et le partage d'information" (Binkley 2006, 59, notre traduction). Il est gratifiant de constater que ces premières interventions dans l'encyclopédie ont également permis de bonifier le contenu des articles. En effet, chacun des ajouts, changements ou modifications d'un article dans Wikipédia peut servir à son tour de catalyseur à d'autres ajouts, 
changements ou modifications. Le plus souvent, le texte concerné se voit amélioré ou bonifié et profite à l'ensemble de la communauté.

\section{UNE «NOUVELLE VISION» : LE PROJET POIRIER}

Convaincu du fort potentiel de l'encyclopédie en matière de transmission du savoir et de la visibilité importante des projets de Wikimédia sur Internet, BAnQ a amorcé un projet de téléversement de photographies au cours de l'été 2014. L'objectif principal était, rappelons-le, de mettre à la disposition des contributeurs des clichés numérisés qu'ils pourraient librement utiliser pour illustrer les articles des différentes versions linguistiques de l'encyclopédie.

Nous souhaitions également nouer des liens et établir une logique de collaboration avec la communauté des contributeurs de Wikipédia, souvent peu informés de l'existence de fonds d'archives pertinents et de la richesse ainsi disponible pour l'encyclopédie comme nous l'a démontré notre première intervention?. Il nous a donc semblé primordial de faire état de nos interventions en toute transparence dès le début du projet. Ce projet représentait de plus un pas significatif en matière de développement d'un esprit collaboratif entre BAnQ et ses partenaires en vue d'élaborer des projets similaires de plus grande envergure.

\section{Comprendre les codes et le fonctionnement de l'univers Wikimédia}

La littérature concernant les activités des GLAM était peu développée en $2014^{10}$. Nous avons donc entrepris une étude des projets menés sur Wikimedia Commons par des institutions fédérales, nationales et municipales telles la National Archives and Records Administration (NARA), la British Library ou les Archives de la ville de Toulouse. Ces comparaisons ont permis d'étudier des stratégies de téléversement différentes selon les institutions et selon les ressources dont chacune d'entre elles dispose. Certaines institutions, telles la NARA ou la British Library ont fait le choix de verser plusieurs dizaines de milliers de documents de différents fonds. D'autres, comme les Archives de la ville de Toulouse ont versé des documents d'un même fonds ${ }^{11}$. Plusieurs organismes ont quant à eux proposé des documents, significatifs à la pièce, issus de différents fonds et collections. Nous avons, à cette étape, fait le choix de concentrer nos efforts sur un unique fonds d'archives.

Il s'est avéré difficile d'obtenir des informations sur les techniques et les outils utilisés pour mener à bien un tel projet puisque peu d'ouvrages font état des interventions des GLAM. Nous avons toutefois cerné l'importance de tisser des liens avec des contributeurs d'expérience afin d'effectuer des actions qui respectent les règles de la communauté. Alors que la NARA est épaulée par un "wikipédien" en résidence ${ }^{12}$, chargé d'alimenter le contenu de l'institution dans les projets wikis, les Archives municipales de Toulouse reçoivent quant à elle l'aide d'un administrateur de Wikimedia Commons, membre de Wikimédia France, en ce qui a trait au volet technique de téléversement de fichiers. Nous sommes pour notre part entrés en contact avec Benoit Rochon, formateur lors des Mardi, c'est Wiki!, chargé de projet et administrateur de Wikimédia Canada, familier des codes et de l'univers Wikipédia. Celui-ci nous a fourni de la documentation spécifique permettant de développer des projets $\mathrm{GLAM}^{13}$ ainsi qu'un appui technique 
pour faciliter nos insertions dans Commons. L'étude comparative des différentes interventions des institutions dans Wikimédia ne nous a malheureusement pas fourni d'informations sur les ressources humaines et matérielles que de tels projets pouvaient nécessiter. Elle a néanmoins été déterminante pour comprendre le fonctionnement de Commons et les différentes stratégies employées par les institutions culturelles.

Étapes primordiales, l'étude du mécanisme de Wikipédia et l'analyse des méthodes proposées en ligne pour ajouter des documents dans Commons nous ont mis sur la voie pour définir le meilleur moyen de répondre aux codes de l'univers Wiki et d'ajouter des documents de manière standardisée. En effet, il était important de s'inscrire en conformité avec ceux-ci pour ne pas risquer de voir tout simplement nos ajouts supprimés par des contributeurs. De plus, ne disposant pas d'outils informatiques ou de robot ${ }^{14}$ permettant d'automatiser le téléversement de masse de photographies dans Commons, comme ce fut utilisé par certaines institutions, ni de l'expertise nécessaire pour élaborer ou repérer un tel outil, nous avons fait le choix de débuter le projet dès l'été 2014 en intégrant un à un les documents dans Commons. Les ressources mises à disposition pour ce projet se matérialisaient par le travail d'étudiantes universitaires, embauchées lors de la période estivale pour renforcer les équipes de la salle de consultation de BAnQ Vieux-Montréal, ainsi que de stagiaires sous la supervision d'un archiviste. La phase de sélection et d'ajout des photographies dans Commons devait prendre en compte le contexte ${ }^{15}$ de travail de l'équipe et s'effectuer de façon simple, pour limiter les erreurs tout en assurant l'ajout des métadonnées et références que nous identifiions comme fondamentales.

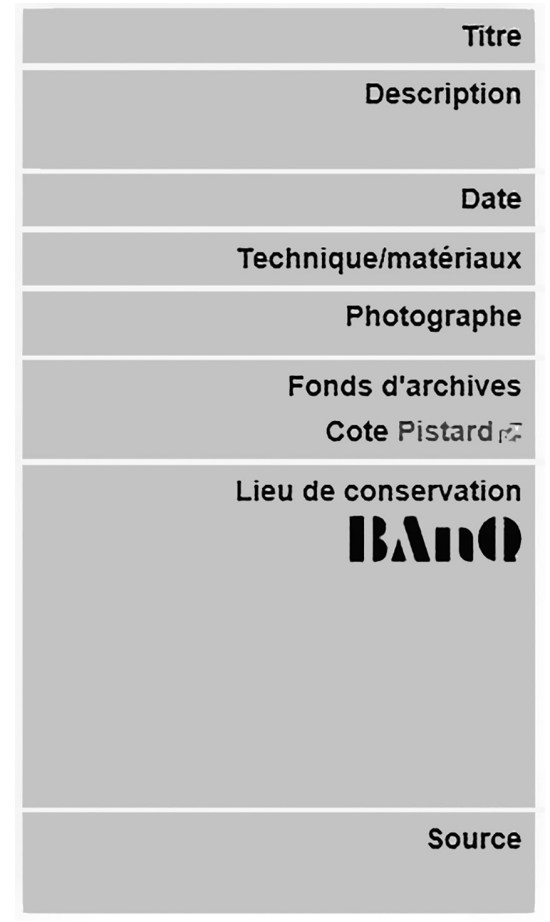

Figure 3 - Métadonnées associées aux images téléversées 
La création d'un modèle de wikicode ${ }^{16}$ normalisé (un canevas type facilitant l'ajout des métadonnées des photographies), dénommé BAnQ-image ${ }^{17}$, simplifiait le processus en intégrant un code répétant automatiquement certaines données et facilitant la mise en page dans Wikimedia Commons. Ce modèle personnalise également les champs visibles dans la zone de description d'une image dans Wikimedia Commons afin de les faire concorder à la réalité du milieu archivistique. Les termes par défaut de l'interface sont remplacés par des termes archivistiques, ID devenant Cote Pistard par exemple. La notion de fonds d'archives, absente des champs normalisés de Wikipédia, était aussi ajoutée. Des modèles particuliers exportant automatiquement les informations concernant l'auteur Conrad Poirier ${ }^{18}$ et l'édifice conservant les documents, BAnQ Vieux-Montréa ${ }^{19}$, étaient également développés. La mention suivante agrémentée d'hyperliens était de plus associée dans les sources de chaque image : "Ce fichier a été numérisé et gracieusement téléversé dans Wikimedia Commons avec l'autorisation et la collaboration de Bibliothèque et Archives nationales du Québec et Wikimédia Canada dans le cadre du Projet Poirier."

Nous avons testé trois techniques proposées par Commons pour téléverser des photographies (à l'exception des outils personnalisés développés par des contributeurs).

La première consiste à utiliser l'assistant d'importation ${ }^{20}$, interface d'ajout simplifiée utile et pertinente pour faciliter le téléversement de photographies sans connaître le fonctionnement du wikicode. L'assistant imagé guide l'usager lors de chaque étape en mettant l'accent sur les notions de droits d'auteur et en fournissant des conseils pratiques pour participer à Commons. Le formulaire standard ${ }^{21}$ de téléversement dans Commons, deuxième outil testé, offre la possibilité d'entrer des informations dans des champs définis sans utiliser le wikicode. Les deux interfaces ne correspondaient pas à nos attentes puisque aucun champ ne permet d'entrer le code du modèle personnalisé.

Nous nous sommes donc tournés vers le troisième outil, le formulaire simple ${ }^{22}$, moins convivial, mais qui offre la possibilité de téléverser un fichier, de le nommer, ainsi que de copier sous forme de wikicode la description du document. Au-delà d'une simple zone de description, l'ensemble du code du modèle comprenant des mentions répétitives comme la licence d'utilisation, l'auteur, le centre d'archives, ainsi que la catégorisation de documents s'inscrivent dans ce champ. Une catégorie peut s'apparenter en archivistique aux termes d'indexation ou aux descripteurs qui facilitent le repérage ou l'appartenance à un ensemble commun. Cette phase d'analyse du mécanisme a d'ailleurs mis en lumière l'importance des catégories dans Commons. Celles-ci identifient des documents ajoutés dans un même projet, par une institution ou par un seul auteur, et offrent un outil de visualisation convivial pour la consultation des photographies. De plus, une page de discussion est créée automatiquement lors de l'ajout d'une nouvelle catégorie, favorisant les échanges avec la communauté. Enfin, nos recherches visant à obtenir des statistiques de consultation des documents versés ont démontré que les outils disponibles comptabilisaient uniquement les données de fichiers de catégories spécifiques. Il était donc primordial pour le projet de créer une catégorie qui serait associée à chaque document afin de pouvoir mesurer l'impact de nos interventions et quantifier le nombre de consultations des photographies.

Ces tests ont finalement servi à la création d'un guide de référence identifiant les critères de nommage des fichiers et les différentes étapes permettant d'aboutir au 
téléversement d'une photographie. Le guide établissait une procédure à suivre pour que concordent les descriptions Pistard des photographies avec le wikicode à ajouter. La réussite du projet reposant en partie sur le nombre d'images téléversées à la fin de l'été, une procédure simple et la plus claire possible facilitait donc d'autant la vitesse de téléversement de l'équipe.

\section{Sélection d'un corpus documentaire}

Nous avons par la suite défini le corpus documentaire qui serait proposé pour l'insertion dans la banque d'images. Trois critères nous ont permis d'identifier un ensemble de documents provenant d'un même fonds d'archives que nous pourrions téléverser. Le premier critère visait à proposer aux contributeurs de Wikipédia un fonds d'intérêt dont les photographies pourraient illustrer des articles variés.

Le deuxième critère consistait à mettre en valeur des photographies déjà numérisées par notre institution puisque près de 9,5 millions de fichiers numériques issus des documents des fonds d'archives sont accessibles à partir du portail Internet de BAnQ. Enfin, le troisième critère était d'ordre juridique puisqu'il était nécessaire d'étudier les règlements en vigueur dans Wikimedia Commons afin de se conformer aux exigences et conditions de téléversement. Celles-ci indiquent: "Tous les fichiers sur Commons (autres que ceux du Domaine Public) doivent être publiés sous licence libre qui autorise n'importe qui à utiliser ces fichiers, pour n'importe quelle utilisation; écrire que "N'importe qui peut réutiliser le travail" ou équivalent n'est pas suffisant. ${ }^{23}$

La question des droits d'auteur est un point sensible pour la plupart des institutions qui ressentent des sentiments ambivalents face à Wikipédia (Ancelle 2015, 14). Si les institutions sont favorables au potentiel de diffusion de l'encyclopédie, elles émettent certaines réserves à l'idée de libérer les droits sur les documents qu'elles conservent, pour tout type d'utilisation y compris commerciale. Dans bien des cas d'ailleurs, les contrats d'acquisition et les ententes conclues avec les donateurs ne donnent pas de latitudes suffisantes aux institutions pour prendre ce type de décision. Face à ce constat, nous recommandons aux archivistes d'amorcer une réflexion au sein de leur institution pour définir une position qu'ils pourront suivre. BAnQ a fait le choix de proposer des documents appartenant au domaine public.

Le choix s'est finalement porté sur le fonds de Conrad Poirier, photojournaliste adepte de la Nouvelle Vision, dont BAnQ conserve 21000 photographies numérisées et décrites à la pièce. Il s'agit d'un témoignage exceptionnel des vies quotidienne, culturelle, intellectuelle, économique, sportive et sociale des décennies 1930 à 1960 à Montréal. L'œuvre de Poirier rend plus largement compte des mutations sociales au milieu du siècle dernier au Québec.

Étant donné le grand nombre de photographies conservées dans le fonds d'archives de Conrad Poirier et souhaitant rester réalistes sur nos capacités à intégrer un nombre aussi important de documents, nous avons établi des critères pour effectuer une sélection de photographies significatives d'intérêt pour l'encyclopédie. Les photographies antérieures à 1949 appartenant donc au domaine public au Canada étaient sélectionnées lorsqu'elles représentaient des lieux identifiés (rues, édifices, villes ou villages), des cérémonies et événements, des activités sportives ou saisonnières, ainsi que des personnalités. 
En présence de plusieurs photographies d'un même événement, la photographie la plus représentative était choisie. Les résultats de ces recherches ont été consignés dans un tableau de suivi comprenant des informations qui permettraient ensuite à l'archiviste responsable de valider le choix des photographies retenues et de compiler des données sur le nombre de documents à intégrer. Afin d'ajouter correctement les documents dans Wikimedia Commons, le lien vers les notices de Pistard devait également servir au repérage rapide des références à indiquer avec la photographie. Au total, 1782 photographies d'intérêt couvrant les années 1933 à 1948 ont ainsi été repérées.

\section{Réalisation et déroulement}

Chaque membre de l'équipe a débuté ses interventions par la création d'un compte sur Wikimedia Commons afin de $s^{\prime}$ identifier ${ }^{24}$, ceci pour pouvoir faciliter le suivi, le repérage des documents versés et les éventuels échanges avec les contributeurs. La nouvelle catégorie nommée BAnQ-Projet Poirier voyait également le jour afin de regrouper les photographies téléversées dans un même ensemble. Après une validation de la sélection par un archiviste, les fichiers numériques ont été ajoutés dans Wikimedia Commons sous des dimensions identiques: format JPEG comme recommandé par la plateforme, taille $8 \times 10$ pouces avec une résolution de $96 \mathrm{ppp}$. Ces dimensions permettent une consultation et une utilisation des documents pour différents usages tout en limitant les utilisations commerciales des fichiers, en conformité avec la position institutionnelle de BAnQ, de par leur résolution peu élevée. L'ensemble des photographies était accompagné du modèle wikicode comportant des métadonnées et des catégories identifiées dans la phase d'analyse. Un lien hypertexte menant vers les notices des documents dans la base de données Pistard était également ajouté dans les descriptions de chacune des photographies. Lors de cette étape, les deux tiers des photographies ont été téléversés par le personnel de BAnQ, le dernier tiers a été pris en charge par Benoît Rochon.

\section{Stratégie de mise en valeur et de promotion du projet}

Une fois les photographies disponibles dans Wikimedia Commons, il était désormais important que celles-ci soient utilisées dans des articles, but premier du projet. Il fallait donc faire connaître nos démarches et l'étendue des documents mis à la disposition des contributeurs. Une page ${ }^{25}$ dédiée au projet Poirier a été créée au début du mois de juillet 2014 sur la page GLAM de BAnQ afin de faire état des avancées et des contributions pouvant être apportées par la communauté. Le lancement, fruit d'une stratégie de communication, fut annoncé le 19 octobre 2014 lors du Mois international de la contribution francophone de Wikipédia, pendant un atelier Wiki à la Grande Bibliothèque. Un article fut publié le même jour sur le blogue de la Fondation Wikimédia et annoncé dans les médias sociaux utilisés par la Fondation. L'information était également relayée sur le compte Facebook de BAnQ et sur la page d'accueil du portail de l'institution. Il est difficile de quantifier la portée de cette annonce dans les médias sociaux, mais nous estimons à $380000^{26}$ le nombre d'abonnés de Twitter qui ont pu voir l'annonce du projet. L'information a été partagée par des institutions nationales à travers le monde telles les Archives nationales de France et traduite sur des sites en plusieurs langues. La communauté des contributeurs du Projet Québec ${ }^{27}$ était 
également informée de nos interventions et les accueillait favorablement. Les intéressés sont depuis appelés à contribuer au projet de façons diverses telles que :

- l'utilisation des photographies pour illustrer des articles de l'encyclopédie, dans toutes ses versions linguistiques;

- la catégorisation plus fine des photographies pour faciliter leur repérage;

- la wikification ${ }^{28}$ des descriptions, c'est-à-dire l'ajout des liens internes à Wikipédia;

- la traduction des descriptions dans d'autres langues.

Les interventions citées sont loin d'être exhaustives, Wikimedia Commons permettant entre autres de géolocaliser des documents ou d'effectuer un travail de restauration numérique sur des documents endommagés, sans pour autant supprimer la version originale. À titre d'exemple, la restauration peut parfois révéler des erreurs de description de documents endommagés par l'usure du temps. L'interprétation d'une photographie prise quelques jours après le massacre de Wounded Knee et conservée par la Library of Congress a été radicalement modifiée suite au travail de restauration de Lise Broer ${ }^{29}$, contributrice de Wikipédia.

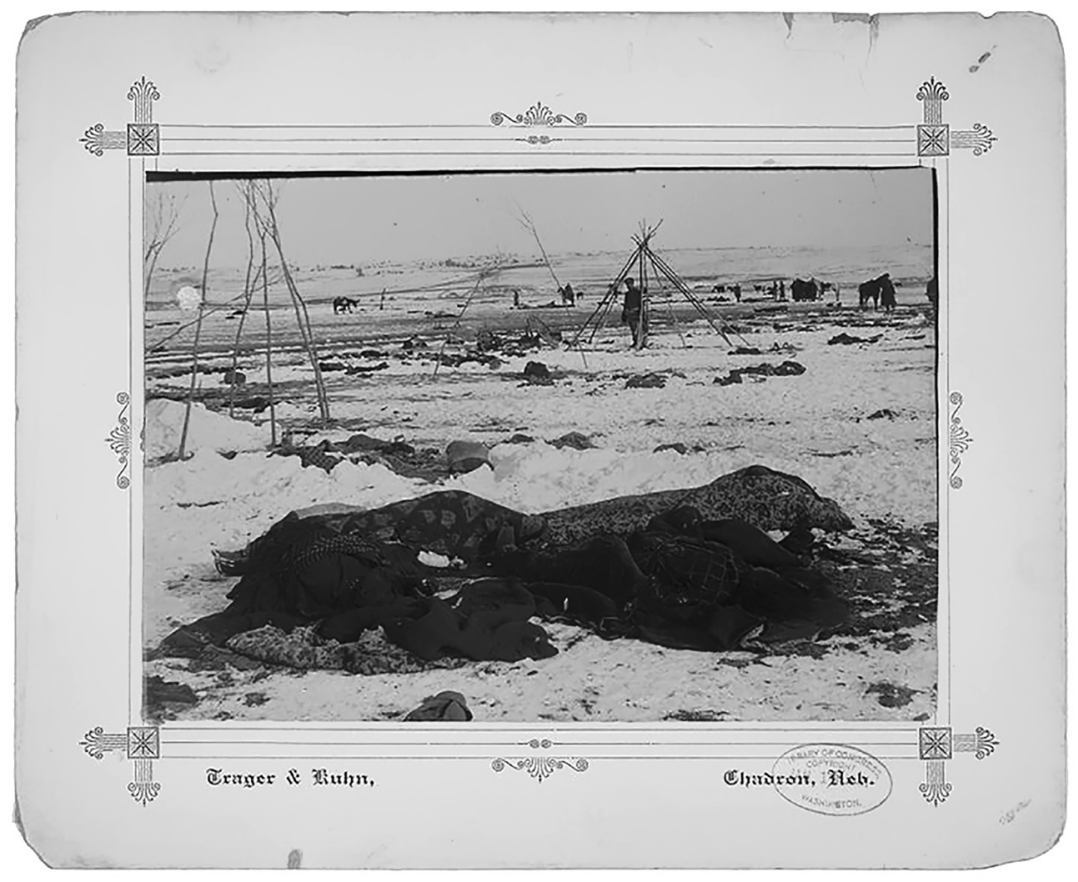

Figure 4 - Big Foot's camp three weeks after the Wounded Knee Massacre. Mislabeled scattered debris of camp. Trager \& Kuhn, Chadron, Nebr.17 janvier 1891. Photographie conservée par la National Archives and Records Administration. Version non restaurée. 


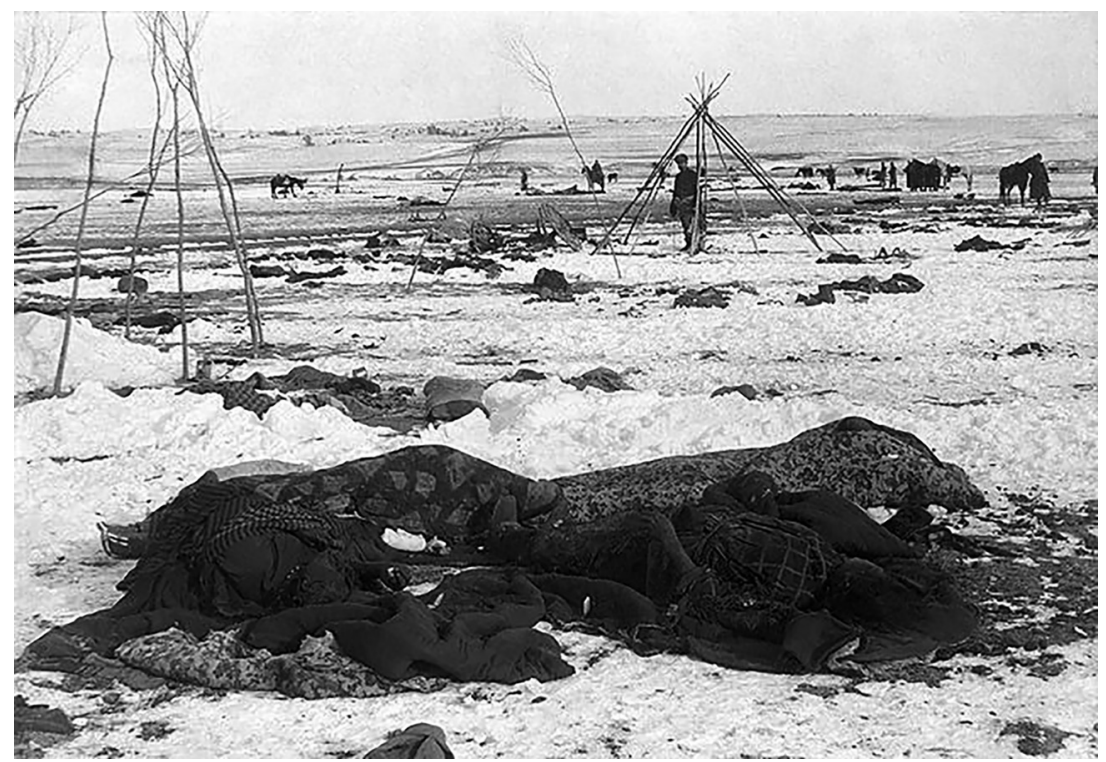

Figure 5 - Suite à la restauration par une collaboratrice de Wikipédia, le titre est désormais: Big Foot's camp three weeks after the Wounded Knee Massacre, with bodies of several Lakota Sioux people wrapped in blankets.

Autrefois décrite avec la mention "Mislabeled scattered debris of camp", celle-ci a été remplacée par "four buman remains in foreground partially wrapped in blankets" après la découverte de corps enroulés dans des couvertures ${ }^{30}$.

La réponse de la Library of Congress suite à ce signalement fut la suivante :

Upon viewing the high-res TIFF file we made of the file, the human remains are quite visible, indeed. Thank you very much for contacting us regarding this image, and for your interest in our collections. You can imagine that among a collection of 14 million items here, there are a lot of secrets waiting to be uncovered!

\section{Résultats}

Les résultats du projet Poirier sont positifs à plusieurs niveaux et l'utilisation des documents dans les articles de Wikipédia dépasse nos attentes. Plusieurs dizaines de contributeurs ont répondu à l'appel de BAnQ et ont utilisé des photographies de Poirier pour illustrer des articles de l'encyclopédie en 127 langues. En un an, les photographies du projet Poirier ont été utilisées dans 1017 articles de Wikipédia. Au total, ceux-ci ont été consultés 29,5 millions de fois par des utilisateurs du monde entier $^{31}$. Cent trente articles illustrés par une photographie de Poirier témoignant de l'annonce de la capitulation de l'Allemagne nazie le 7 mai 1945 ont été consultés 8 millions de fois sur la même période. 


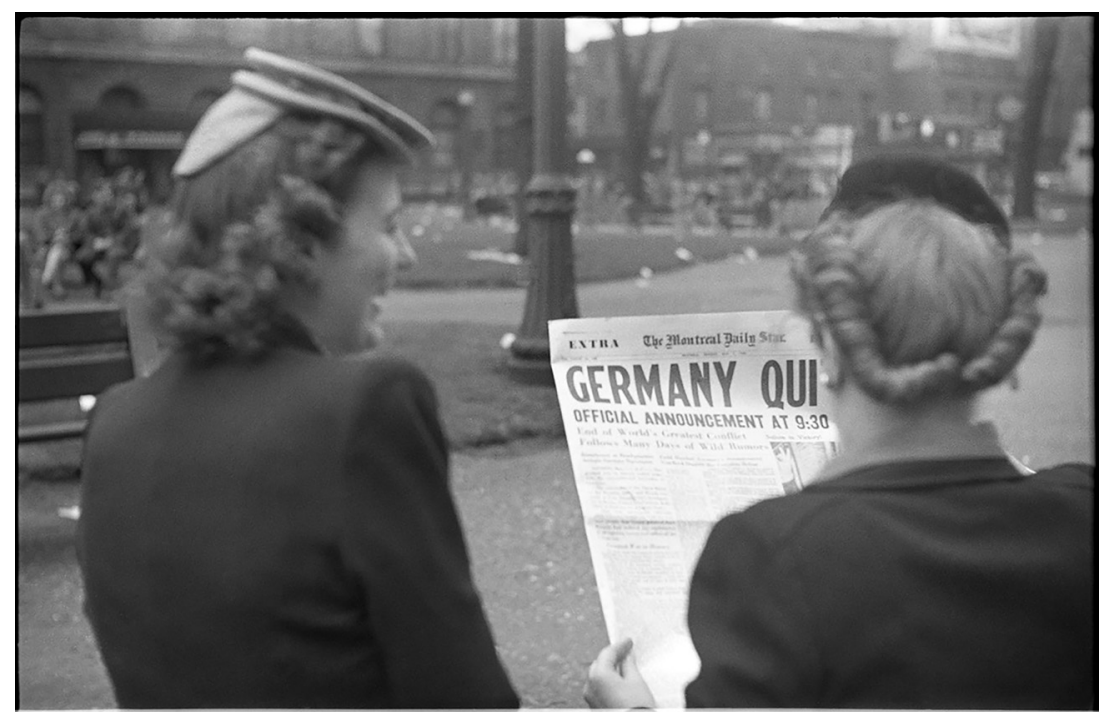

Figure 6 - News. V.E. Day, 7 mai 1945. BAnQ Vieux-Montréal, fonds Conrad Poirier (P48, S1, P12270).

Photo: Conrad Poirier.

Les documents sont également utilisés pour l'élaboration de projets éducatifs et pédagogiques comme c'est le cas du cours Activité thématique en sciences sociales de l'Université du Québec en Outaouais, dans lequel les étudiants sont invités à contribuer à Wikipédia en ajoutant des photographies de Conrad Poirier dans des $\operatorname{articles}^{32}$. D'autre part, si lors de nos attentes initiales nous pensions principalement que les images serviraient à illustrer des articles existants, certains exemples démontrent qu'elles s'inscrivent aussi comme éléments déclencheurs pour la création de nouveaux articles ${ }^{33}$. Wikimedia Commons donne également la possibilité aux contributeurs d'ajouter des informations et de faire des corrections dans les descriptions des photographies, certaines descriptions de document du projet Poirier ont ainsi été bonifiées et corrigées.

Le potentiel de diffusion de Commons en dehors des plateformes de l'univers Wikimédia n'est pas non plus à négliger. Grâce à la qualité de son référencement, les premiers résultats d'une recherche d'image dans un moteur de recherche proviennent généralement de Wikimedia Commons. De plus, de par son essence même favorisant la libre réutilisation des images, Commons attire, telle une banque d'images, un large éventail de journalistes, d'éditeurs ou de professeurs qui souhaitent repérer en peu de temps et à peu de frais un visuel qu'ils peuvent ensuite librement utiliser pour leurs besoins. BAnQ a d'ailleurs reçu depuis le début du projet un plus grand nombre de demandes pour la réutilisation des documents de Conrad Poirier provenant de l'étranger notamment d'Europe. Forte de ses résultats et du succès rencontré par le projet Poirier, BAnQ a démarré d'autres projets collaboratifs sur Wikimédia. 


\section{WIKISOURCE ET LES AUTRES PROJETS DE BANQ DANS L'UNIVERS WIKIMÉDIA}

Depuis, BAnQ poursuit ses interventions dans les plateformes Wikimédia, notamment dans Wikisource. L'institution met à la disposition des contributeurs des ouvrages publiés et des documents d'archives d'intérêt historique pour leur permettre de les transcrire. Cette initiative a pour but de faire découvrir à un plus grand nombre de personnes des événements ou des aspects de l'histoire du Québec par la transcription et la lecture. De plus, elle facilite l'accès à des documents difficiles à lire du fait d'une écriture manuscrite parfois en ancien français, favorise la lecture sur tous les appareils numériques et permet le traitement de l'information par des moyens numériques telle la recherche en texte intégral. Les résultats du volet retranscription de livres sur Wikisource sont tout aussi probants. L'objectif de 2015 était de compléter la transcription de 26 titres. Il a été largement dépassé puisque, parmi les 140 titres provenant des collections numériques de BAnQ téléversés sur le site, 39 ont été transcrits et relus trois fois, puis rendus publics (38 en français, 1 en algonquin). Environ 75 collaborateurs ont participé à la transcription des titres versés par BAnQ. Entre le $1^{\text {er }}$ octobre 2014 et le 30 juin 2015, les 39 titres ont fait l'objet de 15971 téléchargements. Ce qui démontre l'intérêt des internautes. L'ouvrage le plus populaire, 350 recettes de cuisine de $\mathrm{M}^{\text {Ile }}$ Jeanne Anctil, a été retranscrit par la communauté "wikisourcienne" en 48 heures. Téléchargé 1475 fois, il s'agit de l'un des ouvrages les plus populaires de Wikisource.

BAnQ va également de l'avant avec la plateforme Wikimedia Commons et plusieurs projets de téléversement voient le jour. BAnQ Gatineau met à la disposition des contributeurs une centaine de photographies d'édifices religieux de l'Outaouais provenant du fonds du photographe Champlain Marcil. Un millier de photographies de la collection Félix Barrière de BAnQ Vieux-Montréal sera également ajouté dans la banque d'images. En collaboration avec la Fondation Lionel-Groulx, l'institution travaille aussi à la mise en ligne de centaines d'enregistrements radiophoniques des cours d'histoire de Lionel Groulx. Enfin, la diffusion de cartes postales anciennes de différentes régions du Québec est actuellement à l'étude.

\section{CONCLUSION}

L'utilisation par BAnQ des outils Wikimédia n'allait pas de soi avant 2013, mais, une fois leur potentiel bien évalué et la décision prise de les exploiter, BAnQ a ajusté ses pratiques à ce nouvel environnement numérique. Le cadre juridique de diffusion des images a été adapté, tout comme le choix des références et des métadonnées pertinentes. La sollicitation de la participation citoyenne est d'ailleurs fructueuse quand vient le temps de faire connaitre les fonds et les collections, de corriger et d'enrichir les métadonnées des catalogues de BAnQ. En investissant ainsi des plateformes libres de droits, éprouvées par une solide communauté de "wikipédiens", BAnQ bénéficie d'outils robustes et axés sur un usage ergonomique pour le grand public qui peut plus facilement s'approprier les documents patrimoniaux, les faire connaitre à son tour et devenir un champion de la cause des archives. (Charbonneau et al. 2015a, 79) L'approche proactive que BAnQ a entreprise dans l'univers Wikimédia vise à sortir les archives de leurs cadres habituels. Nous partageons à ce sujet pleinement les conclusions de nos collègues suisses. 
Les professionnels [...] doivent impérativement comprendre et connaître les potentialités de ces outils : il en va de leur crédibilité. Car, actuellement, où notre public va-t-il chercher les informations dont il a besoin? Certainement pas dans nos austères catalogues et inventaires d'archives! À nous donc d'occuper le terrain dans ces mondes virtuels, d'être présents là où se trouve notre public potentiel, et si possible l'aider à arriver dans nos collections pour qu'il puisse les exploiter et les valoriser au mieux. (Kern et Böspflug 2015, 3)

Évidemment, cette présence accrue de BAnQ sur Wikimédia a d'importantes retombées pour la visibilité de ses collections. Qu'il soit québécois et souhaite s'approprier des textes et images à caractère historique, qu'il soit étranger et souhaite découvrir l'histoire du Québec par ses documents, le public profite pleinement de documents soigneusement sélectionnés par des archivistes et des bibliothécaires bien au fait du potentiel de leurs collections. Rayonnement local et international de BAnQ et de ses collections : voilà toute la force du Web et de l'univers Wikimédia. Avec une présence accrue des collections archivistiques dans cet univers, ce sont de nouvelles connaissances qui sont générées et relayées; c'est en quelque sorte l'histoire et la culture du Québec qui s'affirment dans l'univers Web collaboratif.

Jamais l'archiviste n'a eu dans l'histoire de la profession autant d'outils et d'espaces à sa disposition pour faire connaître les richesses du patrimoine qu'il conserve. Jamais il n'a eu autant de collaborateurs potentiels pour l'aider dans ses tâches de description, d'indexation et de promotion du savoir. Il est ainsi nécessaire et fondamental de créer des mécanismes permettant de connaître et conserver les informations fournies par les usagers, dans des plateformes tel Wikipédia, mais également dans nos propres outils et bases de données.

Ces projets mettent en évidence un changement de paradigme dans la pratique archivistique contemporaine. L'archiviste doit se doter d'une intelligence collective et d'un esprit collaboratif. (Charbonneau et al. 2015, 236b) Ces valeurs et ces qualités sont non seulement importantes, mais essentielles pour développer une pratique plus flexible et plus ouverte de l'archivistique.

Denis Boudreau Bibliothécaire, Bibliothèque et Archives nationales du Québec.

Florian Daveau Archiviste de référence, responsable des archives iconographiques, Bibliothèque et Archives nationales du Québec.

Frédéric Giuliano Archiviste-coordonnateur, Bibliothèque et Archives nationales du Québec.

\section{NoTES}

1. Nous tenons à remercier les étudiants et stagiaires de BAnQ: Jessica Bouchard, Haydée Carrasco, Judith Dimitri, Émilie Dufour-Lauzon, Audrey Gauthier, JeanPhilippe Gauthier, Virginie Jobin Costa, Marie-France Leclerc, sans oublier Benoit
Rochon de Wikimédia Canada pour leur participation au projet.

2. Selon Alexa Internet, voir https:// en.wikipedia.org/wiki/List_of_most_popular_ websites (Page consultée le 16 novembre 2015). 
3. En deux ans, les 17 ateliers Mardi, c'est Wiki! ont attiré près de 300 participants en salle et à distance.

4. https://commons.wikimedia.org/wiki/ Accueil?uselang=fr (Page consultée le 2 septembre 2015).

5. Selon la classification en vigueur à BAnQ, la classe CLG désigne les archives privées provenant du Centre de recherche LionelGroulx, la classe MSS désigne les fonds et collections d'archives privées provenant de la Bibliothèque nationale du Québec et la classe $\mathrm{P}$ les autres fonds et collections d'archives privées de BAnQ.

6. Notre réponse à ces commentaires et le fil de cette discussion sont disponibles à l'adresse URL suivante: http://fr.wikipedia.org/wiki/ Discussion_Projet:Québec/Archive_37 (Page consultée le 4 décembre 2014).

7. Parcourir au préalable les forums de discussions du projet (Le Forum des nouveaux et Le Bistro - dédiés aux "vétérans") aurait certainement été utile.

8. On parle ici d'ouvrages publiés recensés dans le catalogue IRIS, mais aussi de ressources numériques offertes dans Pistard ou ailleurs sur notre portail.

9. Voir https://fr.wikipedia.org/wiki/ Discussion_Projet:Qu\%C3\%A9bec/ Archive_37\#Fonds_d.27archives Déjà cité. (Page consultée le 31 août 2015).

10. Nous saluons le travail réalisé par nos confrères suisses dans le domaine. GLAM \& Wikimédia, Revue Arbido, 3 septembre 2015. http://www.arbido.ch/userdocs/arbidoprint/ arbido_2015_3_low.pdf.

11. Voir http://commons.wikimedia.org/ wiki/Category :Fonds_Trutat___Archives_ municipales_de_Toulouse?uselang=fr (Page consultée le 27 août 2015).

12. Les "wikipédiens" en résidence sont des "wikimédiens" qui consacrent une partie de leur temps à tisser des liens avec une organisation dont la philosophie se rapproche grandement de celle du mouvement Wikimédia. En plus d'éditer "à l'intérieur des murs" de l'organisation, ils permettent à cette dernière de mieux comprendre et intégrer la communauté pour, éventuellement, permettre à l'organisation d'établir et de maintenir des contacts avec la communauté une fois la résidence terminée. Bien que certains d'entre eux soient bénévoles, les "wikipédiens" en résidence sont généralement compensés financièrement par l'institution qui les reçoit ou par un chapter "wikimédien". Pour en savoir plus Voir http://outreach.wikimedia.org/wiki/ Wikipedian_in_Residence/fr (Page consultée le 27 août 2015).

13. Voir Dossier de présentation à l'attention des institutions culturelles 2011 https://fr.wikipedia.org/wiki/Fichier:Wikim \%C3\%A9dia_-_Dossier_de_pr \%C3 \%A9 sentation_pour_GLAM.pdf (Page consultée le 27 août 2015).

14. Les robots, plus communément appelés bots informatiques, sont des programmes automatisés ou semi-automatisés qui interagissent avec Wikipédia comme le ferait un contributeur. Ils sont utilisés pour réaliser des tâches répétitives de correction orthographique, de modification à grande échelle, de création d'article ou encore pour l'ajout de photographies en lot auxquelles sont rattachées des métadonnées préalablement identifiées. L'usage d'un bot dans l'univers Wikimédia nécessite une autorisation spéciale et des connaissances en programmation informatique.

15. Les étudiantes travaillaient en contact permanent avec la clientèle du centre d'archives en salle de consultation et étaient susceptibles d'être interrompues à tout moment.

16. Le wikicode appelé également wikitexte, utilisé dans l'ensemble des projets Wikimédia, est un langage de balisage informatique simplifié permettant d'éditer du contenu (texte ou image par exemple) et de le mettre en forme.

17. Voir le modèle BAnQ-image http://commons. wikimedia.org/wiki/Template:BANQimage?uselang=fr (Page consultée le 27 août 2015).

18. Voir le modèle Creator: Conrad Poirier https://commons.wikimedia.org/wiki/ Creator:Conrad_Poirier (Page consultée le 27 août 2015).

19. Voir le modèle Institution: BAnQ VieuxMontréal https://commons.wikimedia. 
org/wiki/Institution :BAnQ_Vieux-Montr\% C3\%A9al (Page consultée le 27 août 2015).

20. Voir l'assistant d'importation https://commons. wikimedia.org/wiki/Special:UploadWizard (Page consultée le 27 août 2015).

21. Voir le Formulaire de téléchargement https://commons.wikimedia.org/w/index. php?title=Special $:$ Upload\&uselang=fr (Page consultée le 27 août 2015).

22. Voir le formulaire simple https://commons. wikimedia.org/w/index.php?title=Special: Upload\&uselang=fr\&uploadformstyle=basic (Page consultée le 27 août 2015).

23. Voir https://commons.wikimedia.org/w/ index.php?title=Commons $:$ Licensing/ fr\&uselang=fr (Page consultée le 27 août 2015).

24. Une courte description est associée à chacun des comptes afin d'indiquer à la communauté que le contributeur agit en tant que membre actif du projet Poirier. Un lien vers le compte d'un archiviste est également ajouté dans le but d'effectuer les suivis et de répondre aux questions et commentaires une fois le contrat des étudiantes terminé.

25. https://commons.wikimedia.org/wiki/ Commons:BAnQ? uselang=fr (Page consultée le 29 septembre 2015).

26. Ce chiffre correspond au nombre de comptes et d'abonnés à des comptes ayant tweeté et retweeté l'information entre le 19 et le 21 octobre 2014.

27. La page du projet Québec est le lieu où les contributeurs de Wikipédia intéressés par des sujets concernant le Québec se rassemblent pour discuter des actions à mener et à coordonner au sein de l'encyclopédie. https://fr.wikipedia.org/wiki/Discussion_ Projet:Québec/Archive_41\#Projet_Conrad_ Poirier (Page consultée le 27 août 2015).

28. Voir à ce sujet https://fr.wikipedia.org/wiki/ Aide :Wikification (Page consultée le 27 août 2015).

29. Page personnelle de la contributrice Lise Broer http://en.wikipedia.org/wiki/ User:Durova (Page consultée le 27 août 2015).

30. Voir http://commons.wikimedia.org/ wiki/Commons :Media_restoration et http://commons.wikimedia.org/wiki/ File:Wounded_Knee_aftermath3.jpg (Pages consultées le 27 août 2015).

31. Voir l'outil de statistique Baglama2 http://tools.wmflabs.org/glamtools/ baglama2/\#gid=158\&month=201507 (Page consultée le 27 août 2015).

32. Page Wikipédia présentant le cours Activité thématique en sciences sociales de l'université du Québec en Outaouais. http://fr.wikipedia. org/wiki/Wikip\%C3\%A9dia:Projets_p\%C3\% A9dagogiques/Universit\%C3\%A9_du_ Qu\%C3\%A9bec_en_Outaouais (Page consultée le 27 août 2015).

33. L'article concernant l'hôtel le Chanteclerc de Saint-Adèle fut créé par un contributeur à partir d'une photographie téléversée dans le projet Poirier. Voir http://fr.wikipedia. org/wiki/H\%C3\%B4tel_Le_Chantecler (Page consultée le 27 août 2015).

\section{BIBLIOGRAPHIE}

ANCELLE, Juliette. 2015. Un bref aperçu du régime contractuel dans le contexte des projets Wikimédia. Arbido 3: 14. [En ligne]. http://www.arbido.ch/userdocs/ arbidoprint/arbido_2015_3_low.pdf (Page consultée le 30 septembre 2015).

ARMETT, Barbara et Valerie FORRESTAL. 2012. Bridging the Gap from Wikipedia to Scholarly Sources: A Simple Discovery Tool. College \& Undergraduate Libraries 19, 2-4: 176-188.

BAYTIYEH, Hoda et Jay PFAFFMAN. 2010. Volunteers in Wikipedia: Why the Community Matters. Educational Technology \& Society 13, 2: 128-140. 
BARRON, Paul. 2011. How Google Works. Are Search Engines Really Dumb and Why Should Educators Care? Internet@School Janvier/Février : 12-16.

BINKLEY, Peter. 2006. Wikipedia Grows Up. Feliciter 52, 2 : 59.

CALHOUN, Cate. 2014. Using Wikipedia in Information Literacy Instruction. Tips for Developing Research Skills. College \& Research Libraries News 75, 1: 32-33.

CHARBONNEAU, Normand, Florian DAVEAU, François DAVID et Frédéric GIULIANO. 2015. L'archiviste de référence, de savant à médiateur. In Archiviste de 2030: Réflexions prospectives, sous la dir. de P. Servais. Louvain-la-Neuve, Academia: 73-88.

CHARBONNEAU, Normand, Florian DAVEAU, François DAVID et Frédéric GIULIANO. 2015. La diffusion et la mise en valeur des archives: L'archiviste devenu entrepreneur. In Panorama de l'archivistique contemporaine, évolution de la discipline et de la profession, sous la dir. de L. Gagnon-Arguin et M. Lajeunesse. Québec, Presses de l'université du Québec: 219-237.

DOUCET RAND, Angela. 2010. Mediating at the Student-Wikipedia Intersection. Journal of Library Administration 50, 7-8: 923-932.

DUMOUCHEL, Gabriel et Thierry KARSENTI. 2012. Les compétences informationnelles des étudiants du Québec face au Web: quel bilan?, Conférence présentée au Congrès des milieux documentaires du Québec, Montréal.

ELDER, Danielle, R. Niccole WESTBROOK, et Michele REILLY. 2012. Wikipedia Lover, Not a Hater: Harnessing Wikipedia to Increase the Discoverability of Library Resources. Journal of Web Librarianship 6, 1 : 32-44.

HEAD, Alison J. et Micheal B. EISENBERG. 2010. How Today's College Students Use Wikipedia for Course-related Research. First Monday 15, 3. [En ligne]. http:// firstmonday.org/ojs/index.php/fm/article/view/2830/2476 (Page consultée le 12 janvier 2014).

JACQUEMIN, Bernard. 2012. Autorégulation de rapports sociaux et dispositif dans Wikipédia. Document numérique 14, 3: 57-79.

LALLY, Ann M. et Carolyn E. DUNFORD. 2007. Using Wikipedia to Extend Digital Collections. D-Lib Magazine 13, 5-6. [En ligne]. http://www.dlib.org/dlib/may07/ lally/05lally.html (Page consultée le 2 octobre 2015).

KERN, Gilliane et Katja BOSPFLUG. 2015. Editorial GLAM \& Wikimédia. Arbido 3: 3. [En ligne]. http://www.arbido.ch/userdocs/arbidoprint/arbido_2015_3_low.pdf (Page consultée le 30 septembre 2015).

MATHIS, Rémi. 2011. Défiances et production : Les bibliothèques françaises et Wikipédia. Bulletin des Bibliothèques de France 56, 1: 10-13.

MIRLAND, Cécile. 2012. Usages et pratiques documentaires des jeunes à l'ère du numérique. Bulletin des Bibliothèques de France 57, 4: 78-79.

MURLEY, Diane. 2008. In Defense of Wikipedia. Law Library Journal 100, 3: 593-607.

ROSENZWEIG, Roy. 2006. Can History Be Open Source? Wikipedia and the Future of the Past. The Journal of American History 93, 1: 117-146. 
SNYDER, Johnny. 2013. Wikipedia: Librarians' Perspectives on Its Use as a Reference Source. Reference \& User Services Quarterly 53, 2: 155-163.

SZAJEWSKI, Michael. 2013. Using Wikipedia to Enhance the Visibility of Digitized Archival Assets. D-Lib Magazine 19, 3-4. [En ligne]. http://www.dlib.org/dlib/ march13/szajewski/03szajewski.html (Page consultée le 9 janvier 2014).

WEST, Kathy et Janet WILLIAMSON. 2009. Wikipedia : Friend or Foe? Reference services Review 37, 3: 269-270. 